\title{
Pathological gambling: an overview of assessment and treatment
}

\author{
Sanju George \& Vijaya Murali
}

Abstract Pathological gambling has so far received scant attention in the psychiatric literature. It has a prevalence rate of about $1 \%$ in most countries, and with the deregulation of gambling in the UK the prevalence is set to rise here. Pathological gambling can adversely affect the individual, family and society, and also carries high rates of psychiatric comorbidity. Early identification and appropriate treatment can limit the long-term adverse consequences and improve outcome. This article reviews assessment techniques and tools, and treatment strategies for pathological gambling.

Gambling is a common, socially acceptable and legal leisure activity in most cultures across the world. It involves wagering something of value (usually money) on a game or event whose outcome is unpredictable and determined by chance (Ladouceur et al, 2002). The various types of gambling activities commonly available in the UK are the national lottery, scratch cards, internet gambling, casino games, sports betting, bingo, slot machines and private betting. Results from the most recent British Gambling Prevalence Survey indicate that nearly three-quarters of the adult population had gambled in the previous year and that over half had gambled in the previous week (Sproston et al, 2000). For the large majority, gambling is a recreational activity with no adverse consequences. However, for a significant minority it progresses to pathological gambling, defined in the DSM-IV as 'a persistent and recurrent maladaptive gambling behaviour that disrupts personal, family or vocational pursuits' (American Psychiatric Association, 1994).

The wide array of choices available to the modernday gambler, combined with the deregulation of gambling in the UK, is likely to result in an increase in the number of pathological gamblers and gambling-related problems (Griffiths, 2004). As it is an important public health issue, associated with high rates of psychiatric comorbidity and wideranging personal, family and societal problems, it is crucial that mental health professionals become familiar with this disorder, its assessment and treatment.

\section{Epidemiology}

Pathological gambling typically begins in early adolescence in males (later in females) and runs a chronic, progressive course, punctuated by periods of abstinence and relapses. Although gambling is currently more common among men, the prevalence among women is on the increase. Women are usually older than men when they take up gambling, but once started they develop gambling-related problems more rapidly. In a meta-analysis of 119 prevalence studies, Shaffer et al (1999) found the lifetime and past-year prevalence rates of pathological gambling in adults to be $1.6 \%$ and $1.14 \%$, respectively (adolescents had prevalence rates of $5.77 \%$ and $3.88 \%$, respectively). The British Gambling Prevalence Survey (Sproston et al, 2000) estimated the prevalence of problem gambling in British adults to be $0.8 \%$, and this is likely to increase in the coming years. It is important to note that the prevalence of pathological gambling in psychiatric patients ranges from 6 to $12 \%$.

Adolescents are more vulnerable than adults to gambling and gambling-related problems. Although gambling is illegal for people under 18 years old, surveys have found that nearly three-quarters of adolescents had gambled in the previous year and that rates of problem and pathological gambling in adolescents were nearly twice those in adults. Gambling in this group is strongly associated with alcohol and drug misuse and with depression, and there is some evidence linking early onset of

Sanju George is a specialist registrar in general adult psychiatry and an honorary clinical lecturer in psychiatry at the University of Birmingham (Queen Elizabeth Psychiatric Hospital, Edgbaston, Birmingham B15 2QZ, UK. Tel.: 0121 6782526; e-mail: sanju.george@talk21.com). His main interests lie in undergraduate medical education and pharmacological treatments of drug dependence. Vijaya Murali is a consultant in addiction psychiatry in Birmingham. She has a particular interest in dual diagnosis, and substance misuse in women. 
gambling to more severe later gambling and more negative consequences. Other at-risk populations include minority ethnic groups, those from lower socio-economic groups, and those with mental health or substance misuse problems.

\section{Adverse consequences}

Pathological gambling adversely affects the individual, the family and society. It can negatively influence the gambler's physical and mental health. Gamblers have been noted to report high rates of various psychosomatic disorders and psychiatric problems such as affective, anxiety, substance misuse and personality disorders. Excessive gambling can have a significant impact on the individual's financial situation, often resulting in large debts, poverty and even bankruptcy. To fund their gambling, some resort to criminal activities, ranging from theft and prostitution to violent crime, with obvious legal consequences. Gambling can also adversely affect the gambler's interpersonal relationships and can result in relationship problems, neglect of the family, domestic violence and child abuse (Jacobs et $a l, 1989)$. Children of pathological gamblers have been found to be at increased risk of behavioural problems, depression and substance misuse (Raylu \& Oei, 2001). Costs of gambling borne by society include the cost of the crimes committed by gamblers and the various health and social care costs.

\section{Psychiatric comorbidity}

Research has consistently noted the very high rates of Axis I and Axis II comorbidity in pathological gamblers. People with problem or pathological gambling were many times more likely than the general population to report major psychiatric disorders: major depression, antisocial personality disorder, phobias and current or past history of alcohol misuse (Cunningham-Williams et al, 1998). Depression is probably the most common psychiatric disorder comorbid with pathological gambling. Prevalence figures quoted range from 50 to $75 \%$ (Becona et al, 1996). Two theories have been put forward to explain the relationship between gambling and depression. One is that gamblingrelated losses and other adverse consequences result in depression. The second is that gambling is an activity engaged in to alleviate a depressed state - it is used as an 'antidepressant'.

Suicidal ideation, suicide attempts and completed suicides are much more common in pathological gamblers than in the general population. The rate of suicidal ideation in pathological gamblers has been estimated to range from 20 to $80 \%$ and that of suicide attempts from 4 to $40 \%$. Severe gambling, large debts, coexisting psychiatric disorders and substance use have all been associated with an increased suicide risk.

Black \& Moyer (1998), in a study of 30 pathological gamblers, found that $64 \%$ had a lifetime diagnosis of substance misuse. In a retrospective chart review of 113 pathological gamblers, Kausch (2003) noted that $66.4 \%$ had a lifetime diagnosis of substance misuse or dependence. Other disorders commonly comorbid with pathological gambling are personality disorders, impulse-control disorders, anxiety disorders and attention-deficit hyperactivity disorder. For an excellent overview of psychiatric comorbidity in pathological gamblers see Crockford \& El-Guebaly (1998).

\section{The assessment}

A good assessment will help the clinician to formulate a comprehensive and effective treatment plan. The key areas to be explored are summarised in Box 1. Many gamblers feel ashamed and embarrassed to reveal the true extent of their problems. Hence, the clinician needs to be sensitive and tactful in exploring the individual's gambling behaviour. Sometimes, it might even be appropriate to obtain collateral information from the patient's

Box 1 Summary of key aspects of assessment of the pathological gambler

- Full psychiatric history, including history of presenting complaints, and psychiatric, family, treatment, past and personal histories

- Detailed assessment of gambling behaviour: - initiation

- progression

- current frequency (days per week or hours per day)

- current severity (money spent on gambling proportionate to income)

- types of games played

- maintaining factors

- features of dependence

- Consequences: financial, interpersonal, vocational, social and legal

- Reasons for consultation, motivation to change and expectations of treatment

- Assessment of suicide risk

- Assessment of Axis I and II comorbidity, including substance use disorders

- Comprehensive mental state examination 
partner, spouse or friends (with consent from the patient). It is good to ask the patient to describe in his or her own words the initiation, development and progression of the gambling behaviour in a chronological sequence. The key DSM-IV diagnostic criteria for pathological gambling include preoccupation with gambling, tolerance (the need to wager increasing amounts to achieve excitement), inability to control or stop gambling and 'chasing' one's losses, all of which adversely affect the individual's interpersonal, social and occupational functioning. Features of tolerance, craving, withdrawal symptoms and other diagnostic criteria, if present, will readily confirm the diagnosis, but this forms only part of the assessment.

As maintaining factors can often inform specific interventions, it is important to ask 'What are the reasons why you gamble?' Most commonly reported maintaining factors include negative mood state, boredom and the need to overcome financial problems.

Previous attempts to cut back or quit gambling and treatments tried should inform the clinician in planning the current treatment type and setting. A sensitive exploration of the individual's financial situation (personal and family income and financial stability) and financial problems (gambling debts, bankruptcy) will guide the clinician in suggesting feasible and realistic solutions. The clinician must evaluate the impact of gambling on work (being late, absences, job losses, etc.) and interpersonal and marital life (strained relationships, neglect of family, domestic violence, etc.).

An understanding of a gambler's reasons for consultation will provide indicators of motivation to engage in treatment. A useful question to ask is 'Why are you seeking treatment now?' The person should also be specifically asked about his or her expectations of treatment, in terms of its type, duration and setting.

Despite the high rates of psychiatric comorbidity in pathological gamblers, they often go unrecognised and untreated. A detailed psychiatric history-taking and mental state examination should establish whether there is comorbidity. Gamblers should also be asked about their use/misuse of psychoactive substances and, even more important, their use of alcohol and drugs during gambling sessions.

Assessment of suicide risk (past attempts at selfharm and ongoing suicidal thoughts and plans) forms a crucial part of the overall assessment.

\section{Assessment instruments}

In addition to the clinical interview, several structured instruments have been developed for the screening, diagnosis and assessment of the severity of pathological gambling. The most commonly used
Box 2 Commonly used screening, assessment and diagnostic instruments

- DSM-IV diagnostic criteria: 312.31 (American Psychiatric Association, 1994)

- ICD-10 diagnostic criteria: F63.0 (World Health Organization, 1992)

- The South Oaks Gambling Screen (SOGS; Lesieur \& Blume, 1987)

- The Lie/Bet Questionnaire (Johnson et al, 1997)

- Gamblers Anonymous's Twenty Questions (the GA-20; Gamblers Anonymous, 2005)

of these are listed in Box 2. More recently, many tools have been developed that attempt to assess gamblingrelated attitudes, beliefs, cognitions and urges. These are useful in formulating specific treatments and in monitoring response to treatment.

The clinician has a wide range of instruments to choose from, and the choice should be informed by the population sample, purpose of assessment and the instrument's psychometric properties. It may also be reasonable to use a combination of instruments to capture the complex, multidimensional aspects of gambling.

\section{Aetiology}

A detailed discussion of the various aetiological models of pathological gambling is beyond the scope of this article. Various theories have been postulated: psychoanalytic (unconscious desire to lose, unresolved Oedipial conflicts), learning theories (monetary gain and excitement acting as positive reinforcers), cognitive theories (cognitive distortions such as magnification of one's gambling skills, superstitious beliefs, interpretive biases) and neurotransmitter theories (serotonin, noradrenaline and dopamine dysfunction). To date, no single model fully explains the complex and heterogeneous nature of pathological gambling. The currently preferred approach to its aetiological understanding is eclectic, viewing pathological gambling as the result of a complex interaction between psychological, behavioural, cognitive and biological variables.

\section{Pharmacological interventions Selective serotonin reuptake inhibitors}

Conceptualising pathological gambling as either an impulse-control disorder or an obsessivecompulsive-spectrum disorder implicates the serotonergic system in its aetiology. There is 
also considerable neurobiological evidence to support serotonin (5-HT) system dysfunction in pathological gambling. Hence, fluvoxamine, citalopram, paroxetine, sertraline and fluvoxetine have all been tried with some success in treatment trials for pathological gamblers.

Hollander et al (2000), in a double-blind placebocontrolled study of the use of fluvoxamine (mean dose $195 \mathrm{mg}$ /day) with 15 pathological gamblers, noted significant improvements in the treatment group. However, this study had a small sample size ( 5 of the 15 dropped out) and was of relatively short duration (16 weeks). However, Blanco et al (2002), in a larger and longer study (32 gamblers, 6 months), failed to demonstrate any significant superiority of fluvoxamine over placebo. They also noted a high placebo response rate (59\%).

In a study of 53 pathological gamblers, Kim et al (2002) noted paroxetine to be superior to placebo.

An open-label trial of citalopram with 15 pathological gamblers found considerable improvements on various gambling measures in $87 \%$ of participants (Zimmerman et al, 2002). The therapeutic gains usually occurred in the first few weeks of treatment and were sustained at 12 weeks; they were also found to be independent of the drug's antidepressant effects.

Sertraline was no better than placebo in the treatment of pathological gambling in a double-blind, placebo-controlled study of 60 individuals (Saiz-Ruiz et al, 2005).

\section{Naltrexone}

Naltrexone, a $\mu$-opioid receptor antagonist, is effective in the treatment of a range of impulsive behaviours/disorders such as kleptomania, selfinjurious behaviours and borderline personality disorder. It is also useful in reducing high-urge and craving states in people dependent on alcohol and heroin. Naltrexone's predominant mechanism of action is via the modulation of the mesolimbic dopamine pathway involved in reward and reinforcement. Hence, it is postulated that naltrexone could be used to reduce the rewarding and reinforcing properties of gambling behaviours and thus decrease the urge to gamble.

Kim \& Grant (2001a) treated 17 individuals with DSM-IV diagnosis of pathological gambling for 6 weeks with naltrexone and found significant decreases in gambling thoughts, urges and behaviour. The average dose of naltrexone in this study was $157 \mathrm{mg} /$ day. In a much larger study (83 participants) they noted that $75 \%$ of gamblers treated with naltrexone improved significantly on a range of outcome measures (Kim \& Grant, 2001b). The mean dose of naltrexone was again high (188 mg/day) and only half the sample completed the study. Many participants reported significant adverse effects and many had elevated liver function tests, a particular concern with high-dose naltrexone treatment.

\section{Mood stabilisers}

Some researchers have conceptualised pathological gambling as a bipolar-spectrum disorder, because of shared characteristics such as impulsivity. As the impulsive behaviours in mania are treated effectively with mood stabilisers, it has been suggested that these may also be effective in the treatment of pathological gambling.

Case reports have shown lithium and carbamazepine to be effective in the treatment of the disorder. Pallanti et al (2002) evaluated the efficacy of lithium and valproate in a randomised singleblind study. In all, 15 people on lithium and 16 on valproate completed the 14-week trial. Both groups improved significantly over the trial period (61\% of those taking lithium and $68 \%$ of those taking valproate, with no significant differences in improvement between groups). A more recent study of sustained-release lithium carbonate treatment of a sample of 40 pathological gamblers with bipolar affective disorder found significant improvements in gambling and affective instability in the treatment group compared with placebo (Hollander et al, 2005).

\section{Other drugs}

Other drugs that have been used with some success in treating pathological gambling include olanzapine, bupropion, topiramate and nefazodone (which is no longer licensed in the UK).

\section{Summary of pharmacological interventions}

No drug has been approved for use in the UK or USA to treat pathological gambling and no clear guidelines are currently available. Trials have shown that selective serotonin reuptake inhibitors (SSRIs), naltrexone and mood stabilisers are all effective, although none has demonstrated superiority over others. The existence of comorbidity might often help determine the choice of drug. For example, choose an SSRI if there is coexisting obsessive-compulsivespectrum disorder or depression; choose a mood stabiliser in the presence of comorbid bipolar disorder; and prefer naltrexone if pathological gambling is associated with other impulse-control disorders. Doses of SSRIs and naltrexone required are often at the higher end of the therapeutic range 
and side-effects are therefore more common. As discontinuation studies are lacking, there is no clear evidence on how long to continue treatment: at least 4-6 months initially and then maybe maintenance treatment (Grant et al, 2003). Although empirical evidence is lacking, a combination of pharmacological and psychological therapies might be the best option. More robust studies looking at augmentation strategies, continuation and maintenance treatment and combined pharmacotherapy and psychotherapy are warranted.

\section{Psychological interventions}

\section{Behavioural treatments}

Behavioural theorists view gambling as a learned maladaptive behaviour that can be unlearned through behavioural treatments derived from both classical and operant learning theories.

Much of the early work (in the 1960s) on evaluating behavioural treatments for pathological gambling focused on aversion therapy, which is no longer used. Barker \& Miller (1966) were the first to report the successful use of electrical aversion therapy in a pathological gambler. Seager (1970) found that 5 out of 14 gamblers were abstinent for 1-3 years after aversion treatment. Koller (1972) reported significant improvement in gambling behaviour in 8 out of 12 individuals given aversion treatment. However, some participants in the study also received other interventions, such as attending Gamblers Anonymous.

Other behavioural treatments that have been used successfully include imaginal desensitisation, imaginal relaxation, behavioural monitoring, covert sensitisation and spousal contingency contracting.

McConaghy et al (1983) compared aversion therapy and imaginal desensitisation in 20 pathological gamblers and demonstrated both treatments to be effective. They also noted that the imaginal desensitisation group had significantly lower levels of state and trait anxiety, and fewer gambling behaviours and urges at 1-year follow-up, compared with the aversion therapy group.

In a much larger study $(n=120)$, McConaghy et al (1991) compared four behavioural treatments aversion therapy, imaginal desensitisation, imaginal relaxation and in vivo desensitisation - and found patients receiving imaginal desensitisation to have the best outcome. This study had a relatively longterm follow-up (5.5 years), but the drop-out rate was very high (nearly $50 \%$ ).

Although a range of behavioural treatments have been found to be effective in the treatment of pathological gambling, these days behavioural therapy is more often administered in conjunction with cognitive treatment, as a cognitive-behavioural treatment package.

\section{Cognitive treatments}

Cognitive errors such as gamblers' beliefs about randomness and chance, and the false notion that they can control and predict outcome, play a key role in the development and maintenance of gambling. Cognitive therapy attempts to correct these cognitive errors, which reduces the motivation to gamble.

Ladouceur et al (2001) randomly allocated 66 pathological gamblers to either a cognitive therapy group or a waiting-list control group and demonstrated that $86 \%$ of treatment completers no longer fulfilled the criteria for pathological gambling. They also found that after treatment, gamblers had increased perception of control over the problem and better self-efficacy. These positive effects were maintained at 1-year follow-up. Cognitive therapy has also been found to be effective when delivered in a group format to pathological gamblers.

As already mentioned, in clinical practice cognitive therapy is often administered as part of a cognitivebehavioural package.

\section{Cognitive-behavioural treatments}

These treatments combine cognitive and behavioural aspects and attempt to alter gamblers' cognitions and behaviours. Sharpe \& Tarrier (1993) developed a cognitive-behavioural approach that involves identifying high-risk situations (through functional analysis) or internal and external triggers that lead to urges to gamble and then working on effective coping strategies. Other treatments often incorporated in cognitive-behavioural packages include training in assertiveness, problem-solving, social skills, relapse prevention and relaxation. Specific cognitive-behavioural treatment models have been developed and evaluated by Petry (2002) and Ladouceur et al (2002).

Sylvain et al (1997) evaluated the efficacy of cognitive-behavioural treatment in a sample of 29 male pathological gamblers. The treatment incorporated cognitive restructuring, problem-solving training, social skills training and relapse prevention. Results indicated statistically and clinically significant improvement on many outcome measures and the gains were maintained at 1-year follow-up.

In a randomised study, Echeburura et al (1996) compared four treatments: individual stimulus control and in vivo exposure; group cognitive restructuring; a combination of the two; and a waiting-list control. At 12-month follow-up, the rates 
of abstinence or minimal gambling were $69 \%$ for the individual treatment arm, $35 \%$ for the group treatment and 35\% for the combined treatment. The same research group also evaluated the efficacy of providing a relapse prevention treatment after a 6-week individual intervention (Echeburua et al, 2000). At 12 -month follow-up, less than $20 \%$ of those who received relapse prevention follow-up treatment had relapsed, compared with $50 \%$ of those who received no follow-up treatment.

\section{Gamblers Anonymous}

Gamblers Anonymous is a self-help group modelled on Alcoholics Anonymous. It was founded in 1957 in California and is currently one of the most popular and extensively accessed treatment models for pathological gambling. Gamblers Anonymous uses a medical model of pathological gambling and views total abstinence as the treatment goal. The '12-step recovery program' forms the cornerstone of this treatment and gamblers are assisted in working through steps 1 to 12 by regular attendance at and active participation in group meetings.

It is surprising that despite its popularity, very little research evidence exists to support the efficacy of Gamblers Anonymous. In a study of 232 attendees of Gamblers Anonymous groups, Stewart \& Brown (1988) found abstinence rates of $7.5 \%$ at 1 -year follow-up. They also found that nearly a quarter of new members did not attend a second meeting and nearly three-quarters attended fewer than 10 meetings.

Generally, despite its high rate of attrition, those who regularly attend Gamblers Anonymous groups benefit from this intervention. From a clinical perspective it is more pragmatic to offer Gamblers Anonymous in conjunction with other treatments.

\section{Summary of psychological interventions}

Although a number of psychological interventions are effective in the treatment of pathological gambling, no one approach has clear superiority. Cognitive-behavioural treatments look particularly promising, but results need to be replicated in larger and more representative samples. Major limitations of psychological treatment studies are the lack of long-term follow-up and high drop-out rates. Studies comparing psychological and pharmacological interventions are warranted.

In clinical settings, multimodal treatments often tend to be used. In-patient treatment programmes have not yet been developed widely in the UK (probably because of the resources required to run them), but they are popular in the USA.

\section{Conclusions}

Pathological gambling has so far received scant attention in the psychiatric literature and this field is still in its infancy. With the deregulation of gambling in the UK, the prevalence of pathological gambling is likely to increase in the coming years. It is important to conceptualise pathological gambling as a heterogeneous entity, developed and maintained by a complex interplay of various biological, psychological and social variables. Preliminary research findings offer promising trends in pharmacological and cognitive-behavioural treatments. Improved awareness among health professionals of problem gambling can lead to early recognition and treatment, thus limiting the more severe adverse consequences. Gambling behaviour should therefore be routinely enquired about as part of all psychiatric assessments. Further research is needed to better understand the aetiological mechanisms that would inform effective treatment interventions for this disorder.

\section{References}

American Psychiatric Association (1994) Diagnostic and Statistical Manual of Mental Disorders (4th edn) (DSM-IV). Washington, DC: APA.

Barker, J. C. \& Miller, M. (1966) Aversion therapy for compulsive gambling. Lancet, 1, 491-492.

Becona, E., Del Carmen, L. M. \& Fuentes, M. J. (1996) Pathological gambling and depression. Psychological Reports, 78, 635-640.

Black, D. W. \& Moyer, T. (1998) Clinical features and psychiatric morbidity of subjects with pathological gambling behaviour. Psychiatric Services, 49, 1434-1439.

Blanco, C., Petkova, E., Ibanez, A., et al (2002) A pilot placebocontrolled study of fluvoxamine for pathological gambling. Annals of Clinical Psychiatry, 14, 9-15.

Crockford, D. N. \& El-Guebaly, N. (1998) Psychiatric comorbidity in pathological gambling: a critical review. Canadian Journal of Psychiatry, 43, 43-50.

Cunningham-Williams, R. M., Cottler, L. B., Compton, W. M., et al (1998) Taking chances: problem gamblers and mental health disorders - results from the St. Louis Epidemiological Catchment Area study. American Journal of Public Health, 88, 1093-1096.

Echeburua, E., Baez, C. \& Fernandez-Montalvo, J. (1996) Comparative effectiveness of three therapeutic modalities in psychological treatment of pathological gambling: longterm outcome. Behavioural and Cognitive Psychotherapy, 24, 51-72.

Echeburua, E., Fernandez-Montalvo, J. \& Baez, C. (2000) Relapse prevention in the treatment of slot-machine pathological gamblers: long-term outcome. Behavioural Therapy, 31, 351-364.

Gamblers Anonymous (2005) Twenty Questions. Los Angeles, CA: Gamblers Anonymous. http://www.gamblers anonymous.org/20questions.html

Grant, J. E., Kim, S. W., Potenza, M. N., et al (2003) Advances in the pharmacological treatment of pathological gambling. Journal of Gambling Studies, 19, 85-109.

Griffiths, M. (2004) Betting your life on it. BMJ, 329, 10551056.

Hollander, E., De Caria, C. M., Finkell, J. N., et al (2000) A randomised double-blind fluvoxamine/placebo crossover trial in pathologic gambling. Biological Psychiatry, 47, 813817. 
Hollander, E., Pallanti, S., Allen, A., et al (2005) Does sustainedrelease lithium reduce impulsive gambling and affective instability versus placebo in pathological gamblers with bipolar spectrum disorders? American Journal of Psychiatry, 162, 137-145.

Jacobs, D. F., Marston, A. R., Singer, R. D., et al (1989) Children of problem gamblers. Journal of Gambling Behaviour, 5, 261267.

Johnson, E. E., Hamer, R., Nora, R. M., et al (1997) The Lie/ Bet Questionnaire for screening pathological gamblers. Psychological Reports, 80, 83-88.

Kausch, O. (2003) Patterns of substance abuse among treatment-seeking pathological gamblers. Journal of Substance Abuse Treatment, 25, 263-270.

Kim, S. W. \& Grant, J. E. (2001a) An open naltrexone treatment study of pathological gambling disorder. International Clinical Psychopharmacology, 16, 285-289.

Kim, S. W. \& Grant, J. E. (2001b) The psychopharmacology of pathological gambling. Seminars in Clinical Neuropsychiatry, 6, 184-194.

Kim, S. W., Grant, J. E., Adson, D. E., et al (2002) A double blind, placebo-controlled study of the efficacy and safety of paroxetine in the treatment of pathological gambling. Journal of Clinical Psychiatry, 63, 501-507.

Koller, K. M. (1972) Treatment of poker-machine addicts by aversion therapy. Medical Journal of Australia, 1, 742-745.

Ladouceur, R., Sylvain, C., Boutin, C., et al (2001) Cognitive treatment of pathological gambling. Journal of Nervous and Mental Disease, 189, 774-780.

Ladouceur, R., Sylvain, C., Boutin, C., et al (2002) Understanding and Treating the Pathological Gambler. Chichester: John Wiley \& Sons.

Lesieur, H. R. \& Blume, S. B. (1987) The South Oaks Gambling Screen (SOGS): a new instrument for the identification of pathological gamblers. American Journal of Psychiatry, 144, 1184-1188.

McConaghy, N., Armstrong, M. S., Blaszczynski, A., et al (1983) Controlled comparison of aversive therapy and imaginal desensitization in compulsive gambling. British Journal of Psychiatry, 142, 366-372.

McConaghy, N., Blaszczynski, A. \& Frankova, A. (1991) Comparisons of imaginal desensitisation with other behavioural treatments of pathological gambling. A two- to nine-year follow-up. British Journal of Psychiatry, 159, 390-393.

Pallanti, S., Querciolli, L., Sood, E., et al (2002) Lithium and valproate treatment of pathological gambling: a randomised single-blind study. Journal of Clinical Psychiatry, 63, 559-564.

Petry, N. M. (2002) Pathological Gambling: Etiology, Comorbidity and Treatment. Washington, DC: American Psychiatric Association.

Raylu, N. \& Oei, T. (2001) Pathological gambling: a comprehensive review. JAMA, 286, 141-144.

Saiz-Ruiz, J., Blanco, C., Ibanez, A., et al (2005) Sertraline treatment of pathological gambling: a pilot study. Journal of Clinical Psychiatry, 66, 28-33.

Seager, C. P. (1970) Treatment of compulsive gamblers by electrical aversion. British Journal of Psychiatry, 117, 545553.

Shaffer, H. J., Hall, M. N. \& Vander Bilt, J. (1999) Estimating the prevalence of disordered gambling behavior in the US and Canada: a research synthesis. American Journal of Public Health, 89, 1369-1376.

Sharpe, L. \& Tarrier, N. (1993) Towards a cognitivebehavioural theory of problem gambling. British Journal of Psychiatry, 162, 407-412.

Sproston, K., Erens, B. \& Orford, J. (2000) Gambling Behaviour in Britain: Results from the British Gambling Prevalence Survey. London: National Centre for Social Research.

Stewart, R. M. \& Brown, R. I. F. (1988) An outcome study of Gamblers Anonymous. British Journal of Psychiatry, 152 284-288

Sylvain, C., Ladouceur, R. \& Boisvert, J. M. (1997) Cognitive and behavioural treatment of pathological gambling: a controlled study. Journal of Consulting and Clinical Psychology, $65,727-732$.
World Health Organization (1992) The ICD-10 Classification of Mental and Behavioural Disorders. Clinical Descriptions and Diagnostic Guidelines. Geneva: WHO.

Zimmerman, M., Breen, R. B. \& Posternak, M. A. (2002) An open-label study of citalopram in the treatment of pathological gambling. Journal of Clinical Psychiatry, 63, 4448

\section{MCQs}

1 Aetiological theories postulated to explain pathological gambling include:

a psychoanalytic

b behavioural

c cognitive

d neurobiological

e neurodevelopmental.

2 The diagnostic criteria for pathological gambling incorporate the following:

a tolerance

b preoccupation

c chasing losses

d inability to control the behaviour

e impairment of personal functioning.

3 The following drugs have been found to be effective in the treatment of pathological gambling:

a clozapine

b fluvoxamine

c buspirone

d olanzapine

e carbamazepine.

\section{Pathological gambling:}

a is twice as common in adolescents than in adults

b has an earlier age at onset in females than males

$c$ is more common in minority ethnic groups

$\mathrm{d}$ is more common in upper than lower social classes

e prevalence increases with opportunities to gamble.

5 Behavioural treatments for pathological gambling currently in use are:

a imaginable desensitisation

b aversion therapy

c stimulus control therapy

d covert sensitisation

e spousal contingency contracting.

\begin{tabular}{|c|c|c|c|c|}
\hline \multicolumn{5}{|c|}{ MCQ answers } \\
\hline 1 & 2 & 3 & 4 & 5 \\
\hline a $\mathrm{T}$ & a $\mathrm{T}$ & a $\mathrm{F}$ & a $\mathrm{T}$ & a $\mathrm{T}$ \\
\hline $\mathrm{b} \mathrm{T}$ & $\mathrm{b} \mathrm{T}$ & $\mathrm{b} \mathrm{T}$ & $\mathrm{b} F$ & b F \\
\hline с $\mathrm{T}$ & с $\mathrm{T}$ & c F & с $\mathrm{T}$ & с $\mathrm{T}$ \\
\hline $\mathrm{d} \mathrm{T}$ & $\mathrm{d} \mathrm{T}$ & $\mathrm{d} \mathrm{T}$ & $\mathrm{d} F$ & $\mathrm{~d} \mathrm{~T}$ \\
\hline e $F$ & e $\mathrm{T}$ & e $\mathrm{T}$ & e $\mathrm{T}$ & e $\mathrm{T}$ \\
\hline
\end{tabular}

